\title{
Effect of 3-Day Storage and Temperature on ADP-induced Platelet Aggregation
}

\author{
Nanda Ayu Puspita, ${ }^{1,2}$ Suryawati ${ }^{3}$ \\ ${ }^{1}$ Departement of Biochemistry, Medical Faculty of Syiah Kuala University, Banda Aceh, Indonesia \\ ${ }^{2}$ Biomedical Research Centre, University of Salford, The Crescent, Salford, United Kingdom \\ ${ }^{3}$ Departement of Pharmacology, Medical Faculty of Syiah Kuala University, Banda Aceh, Indonesia
}

\begin{tabular}{|c|c|}
\hline \multirow[t]{3}{*}{ Abstract } & $\begin{array}{l}\text { Objective: To observe platelet functions during the } 3 \text { days of platelet storage } \\
\text { and to evaluate the effect of storage temperature towards platelet aggregation } \\
\text { response. }\end{array}$ \\
\hline & $\begin{array}{l}\text { Methods: Two conditions of platelet storage were used in this study: at room } \\
\text { temperature and in a cold condition at } 4^{\circ} \mathrm{C} \text { to observe the temperature effect } \\
\text { on platelet aggregation. The aggregation test was performed using a } 96 \text {-wells } \\
\text { plate platelet aggregation method. }\end{array}$ \\
\hline & $\begin{array}{l}\text { Results: At room temperature on day } 1 \text {, the response of platelet aggregation } \\
\text { reduced to } 34.9 \pm 10.6 \% \text {, which was less than half of the aggregation of fresh } \\
\text { platelet }(100 \%) \text {. The aggregation on day } 2 \text { reduced modestly }(26.8 \pm 4.2 \%) \text {, } \\
\text { in comparison with that seen on the first day. On day } 3 \text {, platelet aggregation } \\
\text { deteriorated significantly }(6 \pm 1.2 \%) \text {, which was comparable to the control } \\
\text { group without the presence of platelet agonist }(6.1 \pm 1.4 \%) \text {. When platelets } \\
\text { are stored at } 4{ }^{\circ} \mathrm{C} \text {, the aggregation response to ADP stimulation was slightly } \\
\text { different to that shown by platelets stored at room temperature }\left(22^{\circ} \mathrm{C}\right) \text {. At the } \\
4^{\circ} \mathrm{C} \text {, the aggregation response was } 68.11 \pm 34.2 \%, 57.1 \pm 21.4 \% \text {, and } 5.9 \pm 2.6 \% \\
\text { at day } 1 \text {, day } 2 \text {, and day } 3 \text {, respectively. The result showed that storing the } \\
\text { blood at } 4{ }^{\circ} \mathrm{C} \text { or room temperature was not able to preserve platelet function, } \\
\text { as the deterioration of platelet aggregation response over time was still taking } \\
\text { place. Although was not statistically significant, our result indicated that cold } \\
\text { storage reduced platelet responsiveness toward ADP activation. }\end{array}$ \\
\hline $\begin{array}{l}\text { Received: } \\
\text { August } 23,2018\end{array}$ & $\begin{array}{l}\text { Conclusions: This current study provides evidence of the deterioration of } \\
\text { platelet function during first } 3 \text { days of platelet storage. Moreover, we found } \\
\text { that storing platelets in } 4^{\circ} \mathrm{C} \text { showed no significant benefit in preserving the } \\
\text { ADP-induced platelet aggregation capacity compared to that stored in } 22^{\circ} \mathrm{C} \text {. }\end{array}$ \\
\hline $\begin{array}{l}\text { Revised: } \\
\text { September 5, } 2018\end{array}$ & $\begin{array}{l}\text { Keywords: Adenosine diphosphate, platelet, platelet aggregation, platelet } \\
\text { storage }\end{array}$ \\
\hline $\begin{array}{l}\text { Accepted: } \\
\text { September 10, } 2018\end{array}$ & $\begin{array}{l}\text { pISSN: 2302-1381; eISSN: 2338-4506; http://doi.org/10.15850/ijihs.v6n2.1375 } \\
\text { IJIHS. 2018;6(2):73-9 }\end{array}$ \\
\hline
\end{tabular}

\section{Introduction}

In hemostasis, platelet is the most important blood cell. The natural life span of circulating platelets in a physiological condition is up to 8-12 days before they are eventually removed by the resticuloendothelial system. However, the life span will be shorter when platelets are

\footnotetext{
Correspondence:

Nanda Ayu Puspita, Departement of Biochemistry,

Faculty Medicine, Syiah Kuala University

Jl. T. Nyak Arief Darussalam Banda Aceh 23111,

Banda Aceh, Indonesia

e-mail: n.a.puspita@edu.salford.ac.uk
}

taken from the blood vessels for the purpose of platelet transfusion or research. ${ }^{1}$ Due to the complex and time-consuming process of producing platelet concentrates, the time required for blood withdrawal, processing, delivery, and storage before the platelet concentrates are ready for transfusion or research might vary from 1 day to several days. ${ }^{1}$ In the case of clinical purposes, platelet concentrates are generally used after 3-5 days of blood collection, although platelets can be stored longer when the platelet concentrates is stored in plasma in some cases. ${ }^{2,3}$ The length of platelet storage clinically is primarily 
limited by the abundant presence of bacterial contamination and the occurrence of platelet structural lesions (PSLs) after the platelets are stored for 5 days. ${ }^{1,3}$

The potential shelf-life of platelet storage is still debatable until now. ${ }^{1,2}$ Undue platelet activation, platelet membrane protein changes, and bacterial contamination are known as the most commonly found changes occurring on platelets due to storage, resulted in a significant alteration in platelet function..$^{4,5}$ When blood is withdrawn from the blood vessel, platelets are activated by physical stress from the syringe and rapid blood suction, which then leads to minor changes on platelet membrane as well as glycoprotein expression.4,5 During storage, this minor stimulus might be enhanced due to the changes of platelet glycolysis, loss of membrane components, the release of alphagranule contents, and alteration in contractile proteins and metabolic ATP. ${ }^{6}$

As physical stress during blood withdrawal is unavoidable. Also, time and environmental condition during the storage of platelet are also crucial factors that influence platelet deterioration. ${ }^{2}$ Despite the fact that there is an evidence of metabolic and biological activities in platelets after 7 days of storage, platelets have started the morphological and physiological changes from the moment when blood is being withdrawn from the blood vessel. ${ }^{7}$ Several studies have revealed that platelet functions start to deteriorate after 3 days of storage, marked by the occurrence of PSLs, a decrease in platelet mitochondrial activity, increase of reactive oxygen species (ROS) production, and platelet apoptosis. ${ }^{1,8,9}$ However, it is also important to note that, during the first 3 days of blood withdrawal, platelet activity may have also changed, as a response to the exposure to various conditions during storage. ${ }^{2}$ Recent evidence suggests that the temperature of platelet storage is considered as one of the key points in maintaining platelet function. ${ }^{8}$ Many researchers have argued that storing platelets in $4{ }^{\circ} \mathrm{C}$ will prevent bacterial growth and will lower the platelet metabolic rate, although it is still controversial if cold storage gives more benefit in preserving platelet responsiveness to agonist activation. . $^{8,10}$

In this study, the platelet functions during 3 days of platelet storage was evaluated using the platelet aggregation test despite the fact that the light transmission aggregatory (LTA) is widely used as a gold standard for platelet function test. ${ }^{12}$ Platelet aggregation evaluation is considered as the easiest and the most economical method for the purposes of platelet abnormality screening, diagnosis, monitoring therapy, predicting bleeding, and assessing stored platelet. ${ }^{13}$ Two conditions of platelet storage were used in this current study: at room temperature and in a cold condition, in order to observe the effect of environment condition on platelet aggregation. This current study provides evidence of the level of deterioration of platelet function, as the effect of time and temperature during platelet storage.

\section{Methods}

Human Citrated Whole blood was purchased from the National Health Service (NHS) Blood and Transplant, Manchester, United Kingdom (UK) (Application number M061) while Collagen, ADP, adenosine, Prostaglandin-E1, 4(2-Hydroxyethyl) piperazine-1-ethanesulfonic acid, and $\mathrm{N}$ - (2-Hydroxyethyl) piperazine- $\mathrm{N}^{\prime}-$ (2-ethanesulfonic acid) or HEPES as well as Ethylene-bis (oxyethylenenitrilo) tetraacetic acid or ETGA, and apyrase were purchased from Sigma-Aldrich, Dorset, UK. All reagents were purchased from Sigma-Aldrich, Dorset, UK, unless it is otherwise stated.

Citrated whole blood was centrifuged at $1,250 \mathrm{rpm}$ for 15 minutes to obtain platelet rich plasma (PRP). Prostaglandin $\mathrm{E}_{1}\left(\mathrm{PGE}_{1}\right)$ was added at a final concentration of $1 \mu \mathrm{M}$, then incubated at $37^{\circ} \mathrm{C}$ for 10 minutes, followed by subsequent centrifugation at 2,500 rpm for 10 minutes. The platelet pellet was resuspended in a $\mathrm{Ca}^{2+}$ free Tyrode's buffer $(140 \mathrm{mM} \mathrm{NaCl}$, $3 \mathrm{mM} \mathrm{KCl}, 12 \mathrm{mM} \mathrm{NaHCO}, 0.4 \mathrm{mM} \mathrm{NaH} 2 \mathrm{PO}_{4}$, $2 \mathrm{mM} \mathrm{MgCl}_{2}, 5.6 \mathrm{mM}$ glucose; $\mathrm{pH} 6.2$ ) in the addition of $1 \mu \mathrm{M}$ prostaglandin $\mathrm{E}_{1}\left(\mathrm{PGE}_{1}\right)$ and centrifuged at 2,500 rpm for 10 . Platelet pellet was then resuspended in a Tyrode's buffer to give an isolated platelet suspension with a final concentration of 1-3 × 108 cells $/ \mathrm{mL}$. An automated haematology analyser PocH-100i (Sysmex Corporation, Kobe, Japan) was used to count the platelet numbers.

The aggregation test was performed in a 96well plates, according to a method described previously. ${ }^{14}$ As much as $100 \mu \mathrm{L}$ of platelet suspension was transferred into each well of a 96-well microplate. Subsequently, platelet aggregation was induced by the addition of $\mathrm{ADP}$ at a final concentration of $10 \mu \mathrm{M}$. The first reading was taken using a Multiskan $\mathrm{FC}^{\mathrm{TM}}$ microplate photometer with $405 \mathrm{~nm}$ wavelength (Thermo Scientific, Massachusetts, United States). After the first reading, the plate was continuously agitated at $1,000 \mathrm{rpm}$ and subsequent readings were recorded at 1 minute 
intervals for 10 minutes. Platelet aggregation was calculated by subtracting the final reading from the initial reading collected from the same well. In-vitro platelet morphology and aggregates were performed by microscopic observation of a Giemsa-stained thin blood film. Data normalisation was performed by comparing with the maximum response from the corresponding platelet agonists controls.

Statistical analysis was performed using Graphpad prism software version 5.0 from (GraphPad Software, San Diego California USA). Data were presented as mean \pm standard mean error (SEM). Comparison of means was performed using one-way ANOVA with Bonferroni's multiple comparison as a post hoc analysis. P-value smaller than 0.05 was considered statistically significant.

\section{Results}

Morphologically, resting platelets are relatively round and solitary. However, even with the absence of ADP to induce platelet activation, the platelets had developed fine pseudopodia, indicating the early sign of platelet stimulation (Fig. 1). No significant morphological changes on platelets on day 1 , day 2 , and day 3 (Fig. 1).

A preliminary study, ADP was demonstrated to have a potential to induce the aggregation of platelet within the dose range of $1-10 \mu \mathrm{M}$ (data not published). After ADP activation, platelets are activated, leading to the aggregation of platelets. Consequently, single platelets were rarely found amongst the other blood cell population (Fig. 1). This platelet aggregation is marked by the formation of platelet clumps as an ultimate result from platelet activation.

The highest platelet aggregation response was seen on the day of blood withdrawal (Fig. 1). In the absence of ADP, platelet aggregation was not formed, despite the continuous shaking as mechanical stimulation given to the control group. Upon platelet storage, the deterioration platelet aggregation response was seen. On Day 1 , platelet aggregation response reduced more than $50 \%$ when compared to the one in fresh blood (maximum response was $34.9 \pm 10.6 \%$ ). The platelet aggregation on day 2 reduced modestly $(26.8 \pm 4.2 \%)$, in comparison with that seen on the first day. On day 3 of platelet storage, the platelet aggregation deteriorated significantly $(6 \pm 1.2 \%)$, which was comparable to the control group $(6.1 \pm 1.4 \%)$. The result demonstrated the evidence of deterioration of

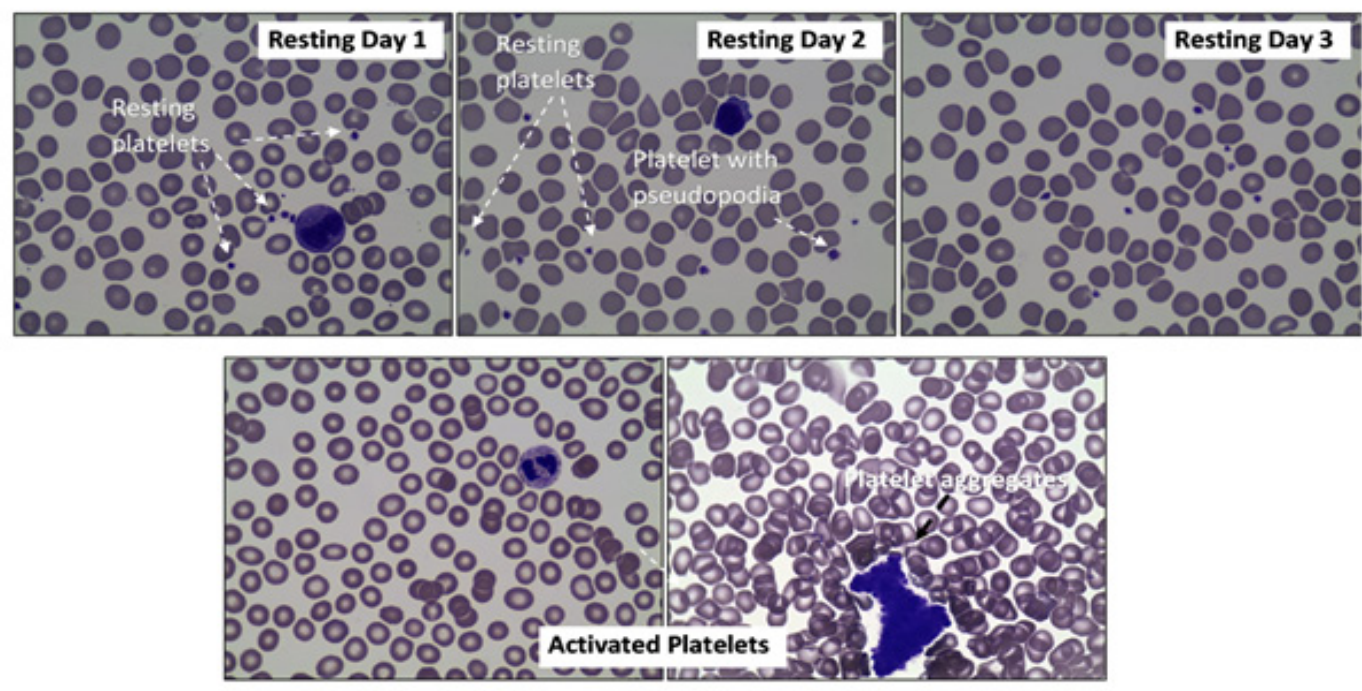

Fig. 1 Microscopic Observation of Platelet Slides. Giemsa-stained Blood Smear (at 400x magnification) from whole Blood. In Resting Condition (Top Figures), Platelets Appeared amongst the other Blood Cells as an Individual Small Fragment. There were No Morphological Changes Observed on Day 1, Day 2, and Day 3. Upon ADP Activation (Bottom Figures), Platelet Population is Markedly Diminished, and Replaced with the Appearance of Platelet Aggregates which Contain a Vast Number of Activated Platelets 


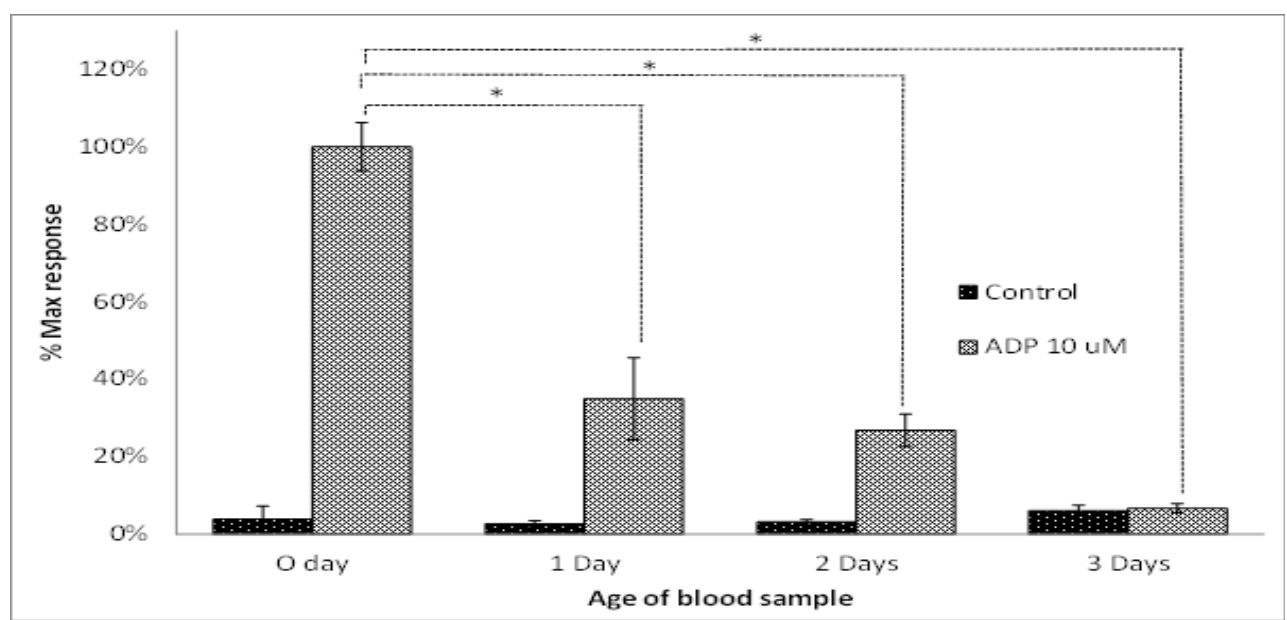

Fig. 2 Maximum Response of Platelet Aggregation over Time. Blood Samples were Kept at Room Temperature $\left(22^{\circ} \mathrm{C}\right)$ and Platelet Aggregation was Induced by ADP at a Concentration of $10 \mu \mathrm{M}$ ). Data on the Graph Represent the Maximum Response of Platelet Aggregation from Four Replicates of the Experiment $(n=4)$ As Means \pm SEM. Statistical Analysis was Performed Using One-way ANOVA with Bonferroni's Multiple Comparison Post-test $(* \mathrm{p}<0.05)$

platelet function during first 3 days of platelet storage.

In order to evaluate the variation of platelet reactivity after ADP stimulation under various external temperature, platelets were kept at room temperature $\left(22^{\circ} \mathrm{C}\right)$ and at $4{ }^{\circ} \mathrm{C}$. Platelet response to stimulation with ADP were slightly different when platelets were stored at $4{ }^{\circ} \mathrm{C}$ or at room temperature for 3 days (Fig. 3). At $4{ }^{\circ} \mathrm{C}$, the aggregation response was $68.11 \pm 34.2 \%$ at day $1,57.1 \pm 21.4 \%$ at day 2 , and $5.9 \pm 2.6 \%$ at day 3 . The result showed that storing the blood at $4{ }^{\circ} \mathrm{C}$ or in room temperature was not able to preserve the platelet function because the deterioration of platelet aggregation response still took place. Although it is not statistically significant, results indicated that cold storage might reduce platelet responsiveness toward ADP activation (Fig. 3).

\section{Discussion}

Platelet concentrates are generally used within a period of 3-5 days due to to the long and time consuming procedure and screening to make sure that the platelets can be used safely. This present study evaluated platelet aggregation response during the first three days of storage, because this period of time is considered as the window period before platelets start to lose their viability. ${ }^{9,13,18}$ Results in this study demonstrate that, after one day of storage, there is a significant deterioration of ADPinduced platelet aggregation response. It is in agreement with previous reports on platelet storage effect on platelet activation, which suggested that platelets have started to lose their activity from the first day of storage. ${ }^{17}$ Platelets only have $45 \%$ activity towards ADP activation on day 1 , and gradually decrease to $12 \%$ on day $5 .{ }^{10}$ Similarly, another study reported that platelet aggregation response towards ADP and collagen activation decrease gradually from day 1 to day 5 , from $78 \%$ to $17 \% \cdot{ }^{16}$ Contrary to those reports, this study observed that platelets have no measurable response to ADP-induced aggregation on day 3 , whilst, from earlier reports, the activity was still measurable until day 5 . Having said that, this study has provided evidence that on day 3 , ADP-induced platelet aggregation activity was similar to that shown by platelets without ADP, which indicates the incapability of platelets to react to ADP activation. Despite evidence of platelet activity after 5 days, to our knowledge the earlier reports have not compared the aggregation activities with activities in the stored resting platelets. Finding in this study is clinically important as, in general, platelet 


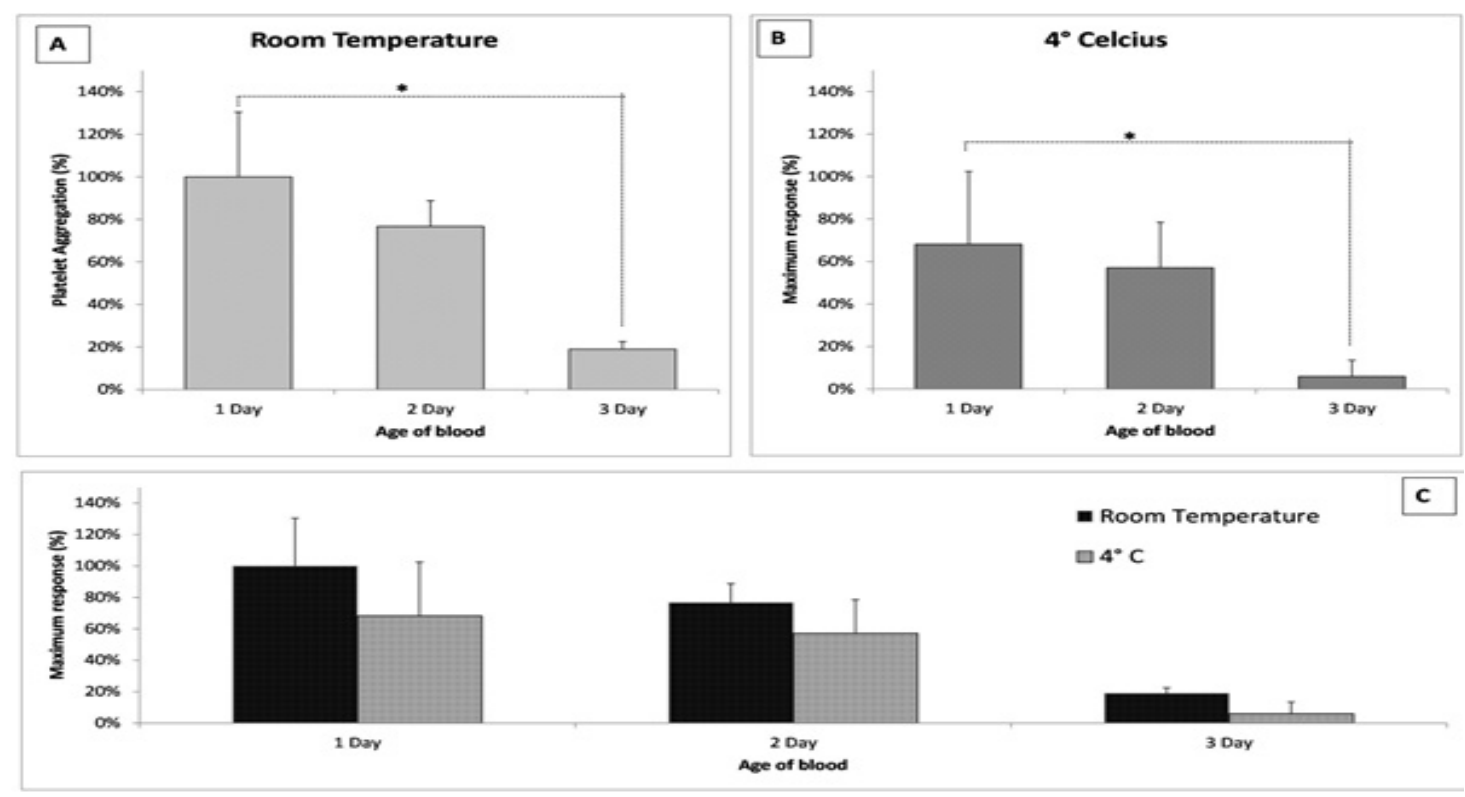

Fig. 3 The Effect of the Temperature on Platelet Aggregation. Platelets were Kept at Room Temperature $\left(22^{\circ} \mathrm{C}\right)$ and $4{ }^{\circ} \mathrm{C}$. Platelet Aggregation Induced by ADP (10 $\mu \mathrm{M}$ ) was Measured on Day 1, Day 2, and Day 3. Data Shown on each Bar Represents the Maximum Response of Platelet Aggregation from Four Replicates of the Experiment $(n=4)$ as Mean \pm SEM. Statistical Analysis was Performed Using Oneway ANOVA with Bonferroni's Multiple Comparison Post-test $(* \mathbf{p}<0.05)$

transfusion takes place after 3 days of storage. ${ }^{9}$ Although progression has been made in terms of platelet storage technology to increase the platelet shelf life, the loss of platelet function during 3 days of storage must be taken into account when providing platelet transfusion to patients with thrombocytopenia in which platelets cannot function.7,18

When stored, platelets show morphological and biological changes. ${ }^{19} \mathrm{~A}$ study has reported the presence of platelet storage lesion (PSL) on stored concentrated platelet. ${ }^{1}$ This platelet storage lesion reflects all deleterious changes in the platelet structure and function starting from the moment blood is drawn from the blood vessel until the platelets are transfused back into the circulatory system. ${ }^{7,18}$ All these tchanges result in the alteration of platelet response to agonist stimulation.,19 After blood withdrawal, the study found that platelets have shown signs of activation, attributed to the mechanical procedure of blood withdrawal. However, the result of this present study does not demonstrate the presence of any significant morphological changes on platelets following 3 days of storage. Despite the limitations of the method used to observe platelet morphology, this study presents further evidence in terms of the deterioration of platelet aggregation response towards ADP stimulation over time. Many researchers have concluded that the deterioration of platelet function is linear to platelet mitochondrial dysfunction, since the initiation and also further events following the activation of platelet are strongly related to the platelet energy metabolism. ${ }^{1,9}$ Most of platelet intracellular ADP are stored in dense granules as a storage ATP, and the remaining ATPs are present in the cytoplasm as metabolic ATP. ${ }^{1}$ The main source of energy is generated through mitochondrial oxidative phosphorylation in platelets, accounting for almost $80 \%$ of total energy in platelet metabolism. In anaerobic conditions, platelets produce energy through cytosolic anaerobic glycolysis, which generates far less ATP than that produced by aerobic metabolism. When the platelet mitochondrial respiration is decreased, platelet intracellular level decrease, leading to the reduction of platelet aggregation capacity. The alteration of mitochondrial capacity of isolated platelet has started on day 1 , followed by a significant 
decrease in respiratory activity on day $2 .{ }^{9}$ This fact is in agreement with the finding in this study which shows that platelet aggregation activity is relatively stable on day 1 and day 2. It is likely that platelet mitochondria is still able to provide sufficient energy for cell metabolism on day 1 , whilst on day 2 when mitochondrial activity started to deteriorate, platelets use storage ATP from dense granules for their metabolism. However, as the ATP pool decreased, platelet activity reduced significantly. Thus, the deterioration of platelet aggregation response is strongly related to the intracellular ATP level. ${ }^{20}$

With regards to the optimum temperature of platelet storage, some researchers have reported that storing platelet at a temperature of $-4^{\circ} \mathrm{C}$ have preserved platelet aggregation potential, compared to $22^{\circ} \mathrm{C}$-stored platelets. ${ }^{4,5}$ Conflicting with an earlier finding, this study shows that storing platelet in $4^{\circ} \mathrm{C}$ fails to preserve ADP-induced platelet aggregation. It is indicated that the aggregation capacity of platelets stored in $4^{\circ} \mathrm{C}$ is similar, if not less, than the platelets stored at room temperature. These isin agreement with past studies conducted by Baimukanova that show the functionality of cold-stored platelets are equivalent to those platelets stored in the room temperature. ${ }^{11}$ Recently, it is well known that that platelets stored in $4{ }^{\circ} \mathrm{C}$ are relatively superior compared to $22{ }^{\circ} \mathrm{C}$ because they exhibited more viable metabolic characteristics, release fewer proinflamatory mediators, hemostatically more effective, has ability to regulate endothelial barrier integrity, and has a more stable clot formation. ${ }^{5}$ In addition this, cold storage also gives benefit in preventing bacterial growth and the occurrence of the PLS during platelet storage. However, in spite of these superiority, there are growing evidence of the occurrence of cold lesion on cold-stored platelet, leading to avcold-induced platelet activation. ${ }^{8}$ Coldinduced lesion is primarily characterized by morphological changes, accompanied by the increase in intracellular calcium level and actin polymerisation degree. ${ }^{8}$ The mechanism of cold-induced platelet activation is not fully understood. ${ }^{8}$ Nevertheless, these drawbacks have raised questions regarding the hemostatic function of platelets stored cold at $4^{\circ} \mathrm{C}$. This study demonstrated that, from day 1 to 3 , the decline of ADP-induced platelet aggregation response is more visible on $4^{\circ} \mathrm{C}$-stored platelet, compared to those of $22{ }^{\circ} \mathrm{C}$-stored platelets. The results indicated that cold-stored platelet function is deteriorating in a same manner as the platelets stored at room temperature. It is important to note that this present study has provided another evidence that cold-stored platelets are not ultimately superior compared to room temperature stored platelets in term of their functionality.

One might argue that the differences of platelet response may be due to the procedure of platelet collection and the aggregation test method. However, recent evidence suggests that there is no difference between platelet collection procedures on platelet activation. ${ }^{16}$ Moreover, this study used plate reader method to measure platelet aggregation response, whilst most of other studies apply standard aggregometer or flow cytometry to measure the platelet aggregation response. The platelet reader method, although not superior to the standard agregometer, gives more benefit in assessing a large number of sample while only using a small amount of platelet sample. ${ }^{14}$ Using the aggregometer and plate reader, the results from platelet agonist-induced platelet aggregation response was qualitatively and quantitatively similar. ${ }^{14}$ Therefore, the effect of the dissimilarity in blood collection procedure and aggregation assay to this contradictory results can be excluded.

In summary, data demonstrated that the platelet functions are seen to be deteriorated significantly during the first 3 days of storage. The study found that platelets have lost more than half of their ADP-induced aggregation capacity on the day 1 , which is likely due to deterioration of platelet mitochondrial activity. On day 3 , the aggregation activity is diminished and comparable to that seen on non-activated platelets, which indicate that platelet have lost their functional in aggregation response. This effect is strongly related to the reduction of intracellular ATP level due to deterioration of platelet mitochondrial activity. Moreover, storing platelets in $4{ }^{\circ} \mathrm{C}$ showed no significant benefit in preserving the aggregation capacity compared to that stored in $22^{\circ} \mathrm{C}$. The study has supported the early consensus in storing platelets, where platelet concentrates shelf life was shorter and must be used immediately in transfusion to avoid the loss of platelet functionality. Furthermore, storing platelets in $22{ }^{\circ} \mathrm{C}$ may improve their availability and avoid the occurrence of cold-induced lesion and coldinduced activation. Nevertheless, additional works are needed for further investigation on storage time and condition, to improve the platelet storage method for clinical use. 


\section{References}

1. Ohto $\mathrm{H}$, Nollet KE. Overview on platelet preservation: Better controls over storage lesion. Transfus Apher Sci. 2011;44(3):321-5.

2. Tynngård N. Preparation, storage and quality control of platelet concentrates. Transfus Apher Sci. 2009;41(2):97-104.

3. Zhang JG, Carter CJ, Culibrk B, Devine DV, Levin E, Scammell K, et al. Buffy-coat platelet variables and metabolism during storage in additive solutions or plasma. Transfusion. 2008;48(5):847-56.

4. Nair PM, Pandya SG, Dallo SF, Reddoch KM, Montgomery RK, et al. Platelets stored at 4 degrees C contribute to superior clot properties compared to current standard-ofcare through fibrin-crosslinking. Br J Haematol. 2017;178(1):119-29.

5. Bynum JA, Adam M, Getz TM, Rodriguez AC, Aden JK, Cap AP, et al. Bioenergetic profiling of platelet mitochondria during storage: $4{ }^{\circ} \mathrm{C}$ storage extends platelet mitochondrial function and viability. Transfusion. 2016;56(Suppl 1):S76-84.

6. Yang J, Yin W, Zhang Y, Sun Y, Ma T, Gu S. et al. Evaluation of the advantages of platelet concentrates stored at $4{ }^{\circ} \mathrm{C}$ versus $22{ }^{\circ} \mathrm{C}$. Transfusion. 2018;58(3):736-47.

7. Thon N, Schubert P, Devine DV. Platelet storage lesion: a new understanding from a proteomic perspective. Transfus Med Rev. 2008;22(4):268-79.

8. Egidi MG, D’Alessandro A, Mandarello G, Zolla L. Troubleshooting in platelet storage temperature and new perspectives through proteomics. Blood Transfus. 2010;8(Suppl 3):73-81.

9. Villarroel JPP, Figueredo R, Guan X, Tomaiuolo M, Karamercan MA, Welsh J, Selak MA, et al. Increased platelet storage time is associated with mitochondrial dysfunction and impaired platelet function. J Surg Res. 2013;184(1):4229.

10. Bakry R, Sayed D, Galal H, Shaker S. Platelet function, activation and apoptosis during and after apheresis. Ther Apheres Dialysis. 2010;15(5):457-64.

11. Baimukanova G, Miyazawa B, Potter DR, Gibb SL, Keating S, Danesh A, et al. The effects of
$22{ }^{\circ} \mathrm{C}$ and $4{ }^{0} \mathrm{C}$ storage of platelets on vascular endothelial integrity and function. Transfusion 2016;56 (Suppl 1):S56-64.

12. Podda GM, Scavone M, Femia EA, Cattaneo M. Aggregometry in the settings of thrombocytopenia, thrombocytosis and antiplatelet therapy. Platelets [serial on the internet] 2018 Mar [cited 2018 Jul 12];14(1):[about 6p.]. Available from:https:// www.tandfonline.com/doi/abs/10.1080/095 37104.2018.1445843?journalCode=iplt20.

13. Michelson AD. Methods for the measurement of platelet function. Am J Cardiol. 2009;103(Suppl 3):S20-6.

14. Vinholt PJ, Nybo M, Nielsen CB, Hvas AM. Light transmission aggregometry using pre-coated microtiter plates and a Victor X5 plate reader. PloS one [serial on the internet]. 2017 OCt [cited 2018 Jan 20];12(10):[about 12p.]. Available from: https://journals.plos.org/plosone/ article?id=10.1371/journal.pone.0185675.

15. Dumont LJ, AuBuchon JP, Whitley P, Herschel LH, Johnson A, McNeil D, et al. Seven-day storage of single-donor platelets: recovery and survival in an autologous transfusion study. Transfusion. 2002;42(7):847-54.

16. Akay $\mathrm{OM}$, Gündüz E, Başyiğit $\mathrm{H}$, Gulbas Z. Platelet function testing during 5-day storage of single and random donor plateletpheresis. Transfus Apheres Sci. 2007;36(3):285-9.

17. Reddoch KM, Pidcoke H, Montgomery RK, Fedyk CG, Aden JK, Ramasubramanian AK, et al. Hemostatic function of apheresis platelets stored at 4 degrees $\mathrm{C}$ and 22 degrees C. Shock. 2014;41(Suppl 1):54-61.

18. Shrivastava M. The platelet storage lesion. Transfus Apheres Sci. 2009;41(2):105-13.

19. Braune S, Walter M, Schulz F, Lendlein A, Jung F. Changes in platelet morphology and function during 24 hours of storage. Clin Hemorheol Microcirc. 2014;58(1):159-70.

20. Diab A, Thomas A, Luban NLC, Wong ECC, Wagner SJ, Levy RJ. Acquired cytochrome C oxidase impairment in apheresis platelets during storage: a possible mechanism for depletion of metabolic adenosine triphosphate. Transfusion. 2012;52(5):1024-30. 